Published in final edited form as:

Semin Roentgenol. 2014 July ; 49(3): 242-254. doi:10.1053/j.ro.2014.10.001.

\title{
PET/MRI - Technical Review
}

\author{
Raymond F. Muzic Jr., PhD ${ }^{1}$ and Frank P. DiFilippo, PhD $^{2}$ \\ Raymond F. Muzic: raymond.muzic@case.edu; Frank P.DiFilippo: difilif@ccf.org \\ ${ }^{1}$ Department of Radiology, University Hospitals Case Medical Center \& Case Center for Imaging \\ Research, Case Western Reserve University, Cleveland, Ohio \\ ${ }^{2}$ Department of Nuclear Medicine, Cleveland Clinic, Cleveland, Ohio \& Cleveland Clinic Lerner \\ College of Medicine, Case Western Reserve University Cleveland, Ohio
}

\begin{abstract}
PET/MR is a hybrid imaging technology with the potential to combine the molecular and functional information of PET with the soft-tissue contrast of MR. Herein we review the technical features and challenges of putting these different technologies together. We emphasize the conceptual to make the material accessible to a wide audience. We begin by reviewing PET/CT, a more mature multi-modality imaging technology, to provide a basis for comparison to the history of PET/MR development. We discuss the motivation and challenges of PET/MR and different approaches that have been used to meet the challenges. We conclude with a speculation about the future of this exciting imaging method.
\end{abstract}

\section{Introduction}

This article is intended to provide an overview of key technical features and challenges associated with combining PET and MR scanning which we will refer to as PET/MR even if it entails coordinated scanning on PET and MR instruments in adjacent rooms. Whereas others have provided excellent coverage of the technical details ${ }^{1-3}$, our goal is to emphasize the conceptual in order to make this material most accessible. Intended to serve a clinical audience, we focus on clinically relevant strategies and systems. We begin by reviewing the history of PET/CT as it provides historical context and a useful reference with which the motivation and development of PET/MR may be considered. We then describe the challenges of combining PET and MR scanning so that readers may understand some of the strategies used in building these systems. Subsequently we turn to specific examples of how manufacturers have applied these strategies to build the systems that do PET and MR scanning sequentially and simultaneously and consider the pros and cons of each approach. We close with a dangerous activity: speculating about the future.

\section{A. Brief history and adoption of PET/CT}

Technical development of combined emission and $\mathrm{x}$-ray tomography dates back to around 1990, when simultaneous SPECT and CT imaging was demonstrated by Hasegawa's

Address for Correspondence. Raymond F. Muzic, Jr., PhD, Department of Radiology, University Hospitals Case Medical Center, 11100 Euclid Avenue, Cleveland, OH 44106, raymond.muzic@case.edu. 
laboratory at UCSF. ${ }^{4,5}$ This pioneering work is remarkable in that it was a fully simultaneous design with a shared detector system. Although this work did not lead to a simultaneous SPECT/CT clinical scanner, it laid the groundwork for sequential hybrid scanners. The same group demonstrated a sequential SPECT/CT system in $1996,{ }^{6}$ which was later commercialized as the GE Hawkeye in $1999 .{ }^{7}$ Around the same time, the first hybrid PET/CT scanner was being developed by CTI PET Systems and tested at University of Pittsburgh. ${ }^{8}$ Shortly afterward, the first commercial PET/CT scanners were introduced in 2001: the GE Discovery LS and the Siemens Biograph. ${ }^{9}$ These and all subsequent commercial PET/CT and SPECT/CT scanners are sequential designs, where the PET or SPECT data are acquired separately from the CT data and where the patient bed has sufficient travel length to cover both fields of view.

Though the technical history of PET/CT is interesting in its own right, the clinical history of PET/CT is even more interesting. When the first commercial scanners were introduced, many questions were raised over clinical benefit versus economics, since the cost of hybrid PET/CT systems was significantly higher than dedicated PET systems. However in about a four year period, dedicated PET went from $100 \%$ to $0 \%$ market share, being completely replaced by hybrid PET/CT in new systems sold ${ }^{9}$ The increased diagnostic accuracy of $\mathrm{PET} / \mathrm{CT}$ compared to $\mathrm{PET}^{10}$ is often identified as a contributing factor to the sudden demand of hybrid systems. The increased throughput of PET/CT may have been a more significant reason, however. The CT scan (less than one minute) replaced the lengthy transmission scan (up to 20 minutes) for attenuation correction. Coincidentally, the emergence of lutetiumbased PET detectors enabled fully 3D image acquisition, reducing emission scan times from 40 minutes to less than 20 minutes. The greatly reduced scan time provided economic justification for hybrid PET/CT and fueled its rapid adoption.

\section{B. Motivation for PET/MR}

The tremendous clinical value and economic success of PET/CT scanning has encouraged the development of PET/MR. Yet, the system complexity and high cost of developing and manufacturing PET/MR scanners are significant barriers to widespread proliferation. An active area of PET/MR research is to identify its added value in order to justify its routine use in a clinical environment. The superior soft tissue contrast of MR along with a suitable PET radiopharmaceutical may make PET/MR preferred for identifying prostate cancer and specifically the portion of the gland or adjacent tissue that is affected so that, for example, radiation therapy treatment can be appropriately focused. The high sensitivity of MR for identifying breast lesions coupled with the high specificity of ${ }^{18}$ F-FDG PET for identifying cancer may lead to PET/MR replacing MR-only scanning in follow-up for x-ray mammographic screening. In particular, PET/MR could be used to guide biopsy and potentially reduce the number of samples needed as well as alleviate patient anxiety by reducing the time interval between a patient receiving a conventional MR scan and a definitive diagnosis. Besides improving sensitivity and specificity, that MR could avoid the ionizing radiation of $\mathrm{CT}$ and yield lower patient radiation exposure with PET/MR than with $\mathrm{PET} / \mathrm{CT}$ is another motivator especially for pediatric patients and patients that undergo repeated scanning. 


\section{C. Brief history of PET/MR development}

Early work towards PET/MR, described in articles published in $1997^{11-14}$, predates the emergence of clinical PET/CT in $2001^{15,16}$. While soft tissue contrast available with MR is generally superior to that with $\mathrm{CT}$, the greater technical challenges of combining MR rather than CT with PET gave PET/CT the inside track for clinical adoption. For reasons to be described in section 2, a fundamental problem to overcome is that photomultiplier tubes (PMTs) do not function within the strong magnetic field required for MRI. Early PET/MR designs entailed a ring of PET crystals inserted within the bore of the MR scanner and coupling the crystals to PMTs via approximately $4 \mathrm{~m}$ long optical fibers ${ }^{14,17}$ so that the PMTs could be placed at a distance from the strong magnetic field. This design was feasible despite the loss of scintillation light within the long optical fibers because of the emergence of new lutetium-based crystals, i.e. LSO, with their five-fold greater light output than the then-state-of-art bismuth germanate ${ }^{18}$ (BGO) crystals. Even though others subsequently built prototype systems using optical fibers ${ }^{19-21}$, the approach lost favor as the light loss degrades the energy resolution and hence system performance. While fibers were obviated by using a type of MR scanner in which the main magnetic field could be turned down during PET scanning ${ }^{22}$, the $0.3 \mathrm{~T}$ low field strength achieved a signal-to-noise ratio that was not clinically satisfactory. As such, none of these approaches has been used in current clinical systems, the design of which is described in subsequent sections in this paper.

With regard to PET/MR systems that have been used to image humans, the first were brainonly prototype imaging systems installed by Siemens for research use in the late $2000 \mathrm{~s}^{23-25}$. The first systems built for clinical use were designed for body imaging. The first Philips Healthcare Ingenuity TF PET/MR ${ }^{26}$ was installed at Mt. Sinai Medical Center in New York as an alpha system in 2009 and then in its clinical configuration in January of 2010. The first Siemens Biograph $\mathrm{mMR}^{27}$ was installed at the Technical University of Munich in November of the same year. GE installed its PET/MR scanner, called by them a PET/CT $+\mathrm{MR},{ }^{28}$ at University Hospital Zurich in 2011. As will be outlined in subsequent sections of this paper, these scanners use different approaches to realize combined PET and MR scanning.

\section{Challenges of PET/MR}

\section{A. PET impacting MR}

MR scanners are built to achieve very uniform strength of the main magnetic field, with homogeneity of typically one part per million for a superconducting magnet ${ }^{29}$, so that the protons resonate at virtually the same frequency. Placing materials in the magnet alters the local magnetic field strength, causing the protons to spin at altered frequencies and causing severe geometrical distortion and susceptibility artifact (signal void). When the "materials" are patients (without implants) this effect is relatively small and could be compensated by proper magnetic field shimming. With materials such as metals that are magnetic (or ferromagnetic as physicists say), the distortion of the magnetic field is large and so too are image artifacts. (In fact, patients with metallic implants face potential safety issues in addition to scan quality issues and must be appropriately screened prior to imaging.) Similarly, adding PET detectors within or near the MR bore degrades magnetic field 
homogeneity and causes image artifacts, particularly if the detectors include magnetic metals. The challenge is then to construct the PET part of the combined scanner in such a way to minimize the impact on the homogeneity of the magnetic field.

Electromagnetic interference (EMI) refers to electrical signals or currents in a circuit that are created by electrical signals flowing in another circuit or being transmitted. When signals occur in the range of frequencies used in radio transmissions, this may be called radiofrequency $(\mathrm{RF})$ noise. (In fact, the resonant frequencies for protons of water in $1.5 \mathrm{~T}$ and $3.0 \mathrm{~T}$ scanners are $64 \mathrm{MHz}$ and $128 \mathrm{MHz}$ and bracket the range of frequencies used by FM radio in the United States.) One type of electromagnetic interference that occurs in everyday life is cross-talk between two telephone lines that run physically near each other. Someone using one line to make a call might hear some of the conversation being carried on the second line. Or, in the case of interference via transmission, a poorly constructed motor or a purposefully created radio jamming signal may interfere with your reception of a TV or radio program. These effects also can occur with PET detectors and all the associated electronics to process counts and have the potential to create signals that are, for example, detected in the MR coils used to create images. Owing to the low-intensity signals that form MR images, the scanner is sensitive even to very low levels of undesired electromagnetic signals.

Beside static magnetic field and RF field, insertion of PET detectors into the MR gantry also compromises the performance of the MR imaging gradient system. A MR imaging gradient is usually built with high spatial linearity and fidelity as well as fast switching time. The presence of additional hardware within the gradient coil significantly alters the MR eddy current characteristics of the system, possibly leading to lengthened switching time and degraded spatial linearity. The gradient imperfection would impact the essential imaging performance, including imaging spatial resolution, scan time and image homogeneity.

\section{B. MR impacting PET}

Photomultiplier tubes (PMT) convert the scintillation light produced by gamma-ray interactions in the crystal into an electronic signal. Specifically, scintillation photons reaching the photocathode at the entrance window of the PMT release electrons. Each such photoelectron, a negatively charged particle, is attracted to the positive voltage toward the back of the PMT in a cascading manner: an electron gains energy as it traverses the electric field between a sequence of electrodes (dynodes) and knocks additional electrons off each, achieving signal amplification. These moving electrons constitute an electric current in the form of a pulse with an area that is proportional to the gamma-ray energy deposited in the crystal, and in the case of a time-of-flight scanner, the pulse timing is sufficiently precise to identify the approximate position of the positron annihilation. The presence of a magnetic field creates a force (Lorentz force) on electrons that is perpendicular to their direction of movement so that the electrons follow a curved trajectory. This defocuses the pulse spatially and temporally so that PMTs do not function properly in the strong magnetic field of an MR scanner.

During the acquisition of MR data, gradient coils are turned on and off in order to spatially encode the MR signal. The associated changes in magnetic field induce electronic signals, 
that is eddy currents, in the PET electronics ${ }^{30}$. The faster and stronger the gradients are, the greater the eddy currents. Likewise, the radiofrequency pulses that tip the protons from their alignment with the main magnetic field may induce high-frequency voltage signals in the PET electronics. Both the gradients and the radiofrequency excitations can interfere with the PET signal analysis that includes detecting a photon, determining its energy, and determining if pairs of detected events meet the energy and timing criteria to be considered a coincidence event that would be attributed to photons created in positron annihilation.

Interestingly, not all the effects of MR on PET are deleterious. In fact, with a strong magnetic field there are (Lorentz) forces that reduce the distance the positively-charged positrons travel before they annihilate. The reduced positron range thus improves the resolution with which the location of radionuclide can be determined ${ }^{31}$. The effect is relatively small with positrons emitted in ${ }^{18} \mathrm{~F}$ decay having a range of $0.5 \mathrm{~mm}$ and potentially significant with positrons emitted in ${ }^{82} \mathrm{Rb}$ decay having a range of $6.1 \mathrm{~mm}^{32}$ in the earth's magnetic field.

\section{C. Attenuation Correction}

The clinical standard for PET images is to correct the data for the absorption of the annihilation photons by the body so that pixel values can be scaled to radioactivity concentration units independent of the depth of the radiopharmaceutical within the body. The initial approach for measuring absorption, and prior to the advent of combined PET/CT scanners, was to perform a transmission scan using a radioactive source outside the body emitting photons of $511 \mathrm{keV}$ or of similar energy. The PET radiation detectors would measure the photons that were transmitted through the body, and a map of the attenuation properties would be reconstructed. Over the course of time various radionuclides and source geometries were considered.

A big change came with the advent of PET/CT scanners wherein an x-ray source and CT detectors were used to make the transmission measurements. This was considerably faster and yielded much less noise compared to a radionuclide source with PET detectors. While development was required to account for the difference in transmission at photon energies used in CT vs. the $511 \mathrm{keV}$ annihilation photons with PET, the technology matured and is in wide-spread use.

In the case of PET/MR scanning, generally the intent is to infer the photon absorption information from MR measurements. This is especially challenging because the underlying physical phenomena that effect MR signals are different from those that impact photon absorption. In PET and CT imaging photon absorption is due primarily to photon interaction with electrons surrounding the atom: inner shell electrons in the case of photoelectric effect and outer shell electrons in the case of Compton scatter. Thus electron density is a shared property underlying photon absorption in PET and CT, and a relatively simple conversion between CT Hounsfield units and PET attenuation factor is feasible. In MR imaging the signal depends on the hydrogen nucleus and its local environment. As such, there is no direct correspondence between the MR signal and photon absorption. In fact, an example that illustrates this and is a current challenge in the field, is that cortical bone and air usually 
have very low MR signal and appear black MR images whereas cortical bone and air represent the extremes in terms of photon absorption within a person.

Although vendors have provided acquisition protocols and data processing that infer photon absorption from MR data, doing so robustly remains an active area of research in order to improve quantification accuracy and reduce artifacts. This will be reviewed in section 3 .

\section{Strategies for PET/MR}

\section{A. Use of MR-friendly materials}

"Primum non nocere" ("First do no harm.") This maxim applies not just to medicine but also to PET/MR design. MR and PET systems that achieve a certain level of performance are expected to maintain their performance in a combined PET/MR system. In other words, the presence of the PET hardware and electronics should not reduce MR imaging performance, and vice-versa. Of course this is not an issue with tri-modality systems with $\mathrm{PET} / \mathrm{CT}$ and MR in separate rooms, assuming that the siting requirements of the individual scanners are followed. For combined PET/MR systems there are several issues with PET and MR compatibility which are discussed in the following sections.

One concern is whether the PET materials affect magnetic field uniformity and MR image quality. Material compatibility is a relatively minor issue with one-room sequential PET/MR. Although the PET gantry contains highly permeable magnetic shielding (mumetal), it is located far enough away that its effect on the MR imaging field of view is easily corrected by shimming. ${ }^{33}$

Simultaneous PET/MR is more of a concern since the PET detectors (crystals and electronics) are located inside the magnet bore and introduces a significant possibility of distorting the magnetic field or gradients. MR compatibility depends on the materials' magnetic susceptibility, the degree to which the material is magnetized when placed in a magnetic field. ${ }^{34}$ Materials having high magnetic susceptibility must be avoided in the PET detectors and integrated electronics. Fortunately the commonly used lutetium-based scintillation crystals (LSO, LYSO) have acceptable magnetic properties. ${ }^{35}$ Even with careful design, some degree of field distortion is inevitable but is corrected effectively by shimming. Measurement of the static magnetic field $\left(\mathrm{B}_{0}\right)$ and the linear field gradient $\left(\mathrm{B}_{1}\right)$ in a commercial simultaneous PET/MR system compared to its base MR-only system demonstrated that the inhomogeneity caused by the PET detectors in integrated PET/MR is negligible. ${ }^{36}$

\section{B. Reducing magnetic fields seen by photomultiplier tubes}

For PET/MR scanners various strategies have been used to minimize the magnetic field in the vicinity of the PMTs to permit them to operate satisfactorily. Examples include turning down the magnetic field when collecting PET data, placing the PMTs at a distance where the field is weak (since field strength significantly diminishes with distance), and shielding the PMTs using a metal alloy (often called mu-metal) that has high magnetic permeability. While turning off the field is not feasible with superconducting magnets used in clinical MR, both distance and magnetic shielding are viable options. For Philips Ingenuity PET/MR with 
its $3 \mathrm{~T}$ magnet, the field strength in the vicinity of the PET detectors, $4.2 \mathrm{~m}$ from the magnet isocenter, would be about $1 \mathrm{mT}$ ( 20 times stronger than the earth's magnetic field).

Although certain multichannel PMTs have been shown to operate in magnetic fields of less than $10 \mathrm{mT}^{17}$, the $1 \mathrm{mT}$ field was still too strong for proper performance of the PMTs ${ }^{26}$ and so it was reduced to about one-tenth of this by adding a sheet of mu-metal to the side of the PET ring that faces the MR system.

\section{C. Using PET detectors that are insensitive to magnetic fields}

PMTs have been used in scintillation (Anger) cameras since their invention by Hal Anger in 1957. The avalanche photodiode (APD) was first considered as an alternative to the PMT in nuclear medicine during the mid 1990s for a preclinical PET application ${ }^{37}$. An APD is an electronic chip that is similar to a PMT, in that both convert scintillation light into an electrical signal with amplification. In a PMT, light releases electrons from the photocathode which are then attracted to and accelerated to the first dynode, a positively-charged plate. Incident electrons there knock off additional electrons that traverse toward the next dynode with the process repeated in a succession of dynodes thereby achieving signal amplification. In an APD there is an "avalanche" of electrons generated inside a semiconductor material. As electrons accelerate through the semiconductor, they cause the emission of more electrons (and electron vacancies called holes which travel in the opposite direction as electrons), thereby amplifying the signal. Approximately a decade after their introduction into nuclear medicine, the relative insensitivity of APDs to magnetic fields was realized with the creation of a PET detector that could operate inside the field of a 7-T MR scanner ${ }^{38}$. Specifically, a $3 \times 3$ array of APDs was used to identify the crystal of a $10 \times 10$ array of LSO in which a gamma ray interacted. APD arrays were subsequently used in the first clinical scanner that could simultaneously perform PET and MR imaging.

\section{D. Reducing PET signal interference with MR}

MR detects small radio frequency (RF) signals and therefore is very sensitive to electromagnetic noise. A MR suite is thoroughly shielded to minimize such interference from the ambient electromagnetic background in a hospital. The presence of PET detector electronics and other supporting electronics within the same room (especially within the same gantry) raises the concern that the PET subsystem may affect MR image quality. Commercial systems are designed and tested to ensure that PET interference with MR is not significant. An initial design strategy for PET/MR is to move as much PET electronics as possible out of the gantry and into an equipment room and to route cabling through appropriate RF filters. However the front-end PET electronics (amplifiers, pulse shaping, digitizing, etc.) must still be located near the scintillation detectors and be shielded.

Sequential PET/MR acquires the PET and MR separately. During MR acquisition, the PET detectors still experience scintillation events from scattered radiation and from natural radioactivity of the lutetium-based crystals. These background events contribute significantly to the MR noise floor, since the events generate noise that is picked up by the RF coils. ${ }^{39}$ To minimize this issue, the high voltage to the PMTs is reduced during the MR scan and is reset to its normal operating value after the MR scan and prior to the PET scan. ${ }^{33}$ 
Simultaneous PET/MR implies that disabling the PET detectors during MR acquisition is not an option. The PET detector modules are covered entirely with copper foil to shield RF noise. Supporting electronics are located outside the imaging field of view adjacent to the magnet and are also covered with copper foil. RF noise in MR images shows no significant difference when measured with the PET detectors powered on or powered off. ${ }^{36}$

\section{E. Reducing MR signal interference with PET}

As noted in section 2, changing electronic or magnetic fields can induce electric currents called eddy currents. In fact, in the Siemens prototype brain PET/MR scanner, fastswitching magnetic gradients were identified as creating eddy currents that distort the PET signals and reduce count rate. Although the count loss was only a maximum of a few percent during a small fraction of the scanning time and a software correction was developed ${ }^{30}$, generally such effects are mitigated by shielding around the PET detectors as well as signal lines. Copper provides good electronic shielding, and in fact, the minor loss in counts was attributed to a shielding defect.

Whereas with PET systems various electronics may be housed within the scanner gantry and image reconstruction computers may even be placed in the scan room, this is not the case with PET-MR and standalone MR systems; MR systems in general are more sensitive to noise than PET systems. As such, while the PET component of the Philips Ingenuity TF PET/MR is similar to the PET component of the Philips Gemini TF, in the PET/MR configuration many of the electronics are relocated to an electrically shielded cabinet placed in the MR equipment room. Even greater separation is used with the GE tri-modality PET/CT+MR with the PET/CT Discovery 690 and Discovery 750w MR systems located in completely separate rooms.

\section{F. Attenuation correction}

As noted previously, determining the photon attenuation of the PET gamma rays from the MR data is nontrivial owing to the fundamentally different underlying physical processes of PET and MR. Note that the "tri-modality" approach to PET/MR, namely the use of individual PET/CT and MR scanners along with a docking shuttle bed for transporting the patient, does not experience this issue since CT-based attenuation correction is available. However the other PET/MR approaches do not generate co-registered CT images, and MRbased attenuation correction must be developed with reasonable accuracy. Currently Philips and Siemens use segmentation approaches, analogous to color-by-numbers. One approach is to assign image voxels to two tissue classes in addition to air, namely lung and soft tissue, based on an MR image. Another approach is to assign voxels to three or more tissue classes by adding bone and/or differentiating soft tissue into fat and lean tissue. The tissue type or segment of each pixel may be determined by (1) matching the patient's MR scan to images in a library of scans taken in other subjects, by (2) using image processing based on anticipated shapes of various organs, or by (3) specialized MR scan sequences to identify tissues by type. Although the library-matching approaches use registration with some including stretching or warping transformations, human anatomy and body composition differ across individuals so this can be unsatisfactory. Likewise, in the second method, determining the shape of an organ such as lung based on typical lung shapes is problematic

Semin Roentgenol. Author manuscript; available in PMC 2015 October 18. 
because of shape differences between individuals and is further complicated by differences in tissue density across the lung and in a way that depends on the lung orientation relative to gravity and occurrence of emphysema or other diseases. Further, most of the early development of the methods was based on normal volunteers with normal anatomy. On top of all this, implants (and attendant artifacts on MR images), abnormal anatomy, and pathological conditions common in clinical patients make the first two methods insufficiently reliable for routine clinical use.

The direction of current research on attenuation correction methodology is toward specialized MR acquisition sequences that can identify different tissue types within individual patients with few a priori assumptions about expected shape or composition. For example, ultra short echo time (UTE) sequences can be used to image cortical bone $e^{40-42}$. With other than the specialized UTE sequences, the MR signals of cortical bone and that of air are quite low and these regions appear as black in images, Figure 1. The bone signal is low because the $\mathrm{T} 2 *$ (spin-spin relaxation in the presence of field inhomogeneity) is so fast that the signals dephase before any conventional MR sequence collects the data. In contrast, the signal from air is essentially noise since air has few hydrogen nuclei. Since these two tissues lie at the extremes of photon attenuation in clinical PET imaging, differentiating the tissues is critical for PET quantitative accuracy. With UTE imaging, the TE times are so short, on the order of 100 microseconds or fractions thereof, such that bone signal has not completely dephased, and bone appears as gray, Figure 1b. Using a dual-echo UTE acquisition, data are also collected with a longer TE wherein bone signal is largely absent and signal from all other tissues is essentially the same as the first echo. Images from the two echoes may then be analyzed to produce an image that indicates bone, soft tissue, and air (see Figure 1c).

Another specialized MR sequence may be used to differentiate fat and lean tissue ${ }^{40}$. As these tissues have relatively similar attenuation at the energy of the PET annihilation photons, the impact on attenuation correction is less than that of identifying bone. On the other hand, these acquisitions can be performed quite rapidly and the ability to localize abnormal FDG uptake to fat and lean tissues has clinical value. In brief, this may be accomplished by using the two-point Dixon method of $\mathrm{MR}^{43}$, Figure 2 . Hydrogen nuclei in the vicinity of fat and water precess or rotate at slightly different speeds. MR data are acquired at two times: one with the fat and water spins aligned or in-phase (IP) and one with these spins 180 degrees apart or in opposed-phase (OP). In the IP image, signal in a pixel equals that of water plus fat $(\mathrm{IP}=\mathrm{W}+\mathrm{F})$ whereas in the $\mathrm{OP}$ image it equals water minus fat $(\mathrm{OP}=\mathrm{W}-\mathrm{F})$. Thus, the IP and $\mathrm{OP}$ images may be added and subtracted to yield images of water and fat $(\mathrm{IP}+\mathrm{OP}=2 \mathrm{~W}, \mathrm{IP}-\mathrm{OP}=2 \mathrm{~F})$, Figures $2 \mathrm{a}$ and $2 \mathrm{~b}$.

\section{Sequential Systems}

\section{A. PET and MR standard systems with software fusion}

Just as software fusion of PET and CT images was the ancestor to and even motivated the emergence of combined PET/CT scanners, the same can be said of software fusion of PET and MR images relative to PET/MR. Software fusion of images acquired using singlemodality scanners is less expensive than using multimodality systems and is more accessible 
owing to the number of single-modality scanners. Software fusion is quite effective for rigid structures. Automated registration algorithms are used routinely to fuse MR and PET brain images. However for deformable structures, such as in the abdomen or head-neck regions, software fusion is much less effective. Use of positioning aids can improve the consistency of software fusion, but logistics and inaccuracies motivate the adoption of more integrated systems.

\section{B. PET/MR systems with PET and MR gantries in different rooms}

Currently there is one commercially available PET/MR system that houses the PET and MR components in separate rooms ${ }^{28}$. In the spectrum of PET/MR scanning systems this GE PET/CT+MR system, Figure 3a, has the greatest simplicity, entailing standard PET/CT and MR systems, respectively PET/CT Discovery 690 and Discovery 750w MR. Further, availability of CT data avoids the challenge of determining attenuation data from MR data. The unique feature of this system is a special trolley arrangement and coils that enable a patient and scanner table-top to be shuttled from the PET/CT to the MR system. The concept of separate scanners with a movable docking patient bed was first demonstrated for SPECT $+\mathrm{CT} \cdot{ }^{44}$ Extending this concept to PET/CT+MR presented new technical challenges, namely the development of a MR-compatible bed and the development of "glove coils" into which the patient slides (in the same manner that the hand slides into a glove).

\section{C. PET/MR systems with separate gantries in the one room}

The Philips Ingenuity TF PET/MR, Figure $3 b$, is currently the only combined PET/MR system designed with separate gantries that are housed in the same room ${ }^{26}$. The system is essentially a combination of modified versions of a Philips Gemini TF PET scanner and a Philips Achieva 3T MR scanner installed in a single room. There is a single patient table located between the gantries. The table sits on a turn-table that can rotate the patient to be head-first (or feet-first) for both PET and MR and has a range of motion (extended compared to dedicated MR systems) that enables whole-body imaging. Moving a patient from being positioned in one gantry to the other takes approximately a minute and can be done without any manual intervention by the technologist.

\section{Simultaneous Systems}

The Siemens Biograph mMR, Figure 3c, is the first clinical simultaneous PET/MR scanner ${ }^{27}$. Its design is based on a prototype brain PET insert for a clinical MR scanner developed by Siemens for research. ${ }^{24,45}$ The success of this prototype design and the engineering knowledge gained led to the fully integrated whole body scanner. The Biograph mMR is based on the Verio 3T MR platform which is modified to include the PET detectors, electronics, cooling system, and other supporting hardware. The PET detectors are constructed as blocks containing an $8 \times 8$ array of LSO crystals being read by a $3 \times 3$ array of APDs. The block detectors are situated inside the magnet, between the gradient coils and RF body coil. With the covers on, the Biograph mMR appears virtually identical to a conventional MR system, with the exceptions that the bore length is slightly longer to account for PET electronics adjacent to the magnet coils and that the patient bed and coils are modified to reduce attenuation of $511 \mathrm{keV}$ photons. 


\section{Pros and Cons of the Scanner Designs}

\section{A. Field of view}

Field of view is an essential consideration in PET and MR design, since it is the primary factor underlying the cost of the scanner. A larger PET field of view necessitates more crystals, PMTs or APDs, and electronic readout channels, adding significantly to the cost of the scanner. A larger MR field of view means more highly uniform magnetic field, which requires more magnet wiring, larger cryogenic system, and larger gradient and shim coils. In brief, bigger scanners are more costly.

The higher cost of a larger scanner has to be weighed against the clinical advantages of a larger scanner. A large field of view is desirable in a clinical environment. A large axial field of view reduces scan time for whole-body PET and MR imaging, and an added bonus is that PET count sensitivity improves as the patient is more completely surrounded by detectors. A large transaxial field of view facilitates imaging obese patients and reduces issues with patient compliance (claustrophobia, discomfort, etc.). A large transaxial field of view permits arms-up imaging, reducing PET attenuation and minimizing MR-AC edge truncation. The clinical desirability is reflected in the market trend from $60-\mathrm{cm}$ bore diameter in dedicated PET and MR scanners to 70-cm bore diameter in the newest MR scanners and nearly $80-\mathrm{cm}$ bore diameter in some PET/CT scanners.

A clear advantage of sequential PET/MR is that the respective PET and MR fields of view are preserved. In contrast, a simultaneous PET/MR with the PET detectors within the MR ring requires an overall reduction in usable field of view diameter. For example, the Siemens Biograph mMR scanner is based on the Verio 3T MR scanner with 70-cm bore diameter. The PET detector modules and associated packaging and shielding are about $5 \mathrm{~cm}$ thick, thus reducing the PET/MR bore diameter to $60 \mathrm{~cm} .{ }^{36}$ The smaller bore diameter is a clinical limitation, and it also is an economic limitation since it uses a more costly $70-\mathrm{cm}$ magnet. About $27 \%$ of its highly uniform magnetic field region is not utilized for imaging but still must be purchased.

\section{B. Scanning time}

"Sequential" means sequential. That is, the total scan time of a PET/MR study is the sum of the scan times of the PET and MR studies, plus the time associated with moving the patient from the MR to PET field of view. For the one-room design (Philips Ingenuity), the additional time for the bed to retract, rotate, and extend is under a minute. For the two-room design (GE PET/CT + MRI), the additional time can be considerably longer depending on proximity and logistics.

"Simultaneous" scanning is truly simultaneous only when the PET and MR fields of view exactly coincide. The degree of simultaneity depends on the particular clinical protocol. This is particularly so with whole-body imaging, where the patient bed is stepped axially to acquire PET and MR data over a specific time interval per bed position. Simultaneous scanning is most efficient if the PET and MR scan periods match, since if the PET scan per bed position is significantly longer than the MR scan per bed position, or vice-versa, then one is idle while it waits for the other to complete. A logical approach is to specify the PET 
scan time per bed position to achieve the required count statistics and to fill that time with appropriate MR whole-body pulse sequences (for example Dixon MR-AC followed by T1weighted and single shot turbo spin echo sequences). A localized diagnostic MR study may also be acquired at a single bed position but is not simultaneous with the whole-body PET study. The time-line of an example of a simultaneous PET/MR study is shown in Figure 4. Many other scan protocols are possible, and a full discussion of PET/MR study designs is found in the literature. ${ }^{46}$

\section{C. Time-of-flight PET}

Time-of-flight (TOF) PET refers to detection of positron annihilation photons with extremely high temporal accuracy to further improve PET image quality. (Note that TOF PET is not to be confused with time-of-flight MR imaging, in which a MR angiography study is acquired by employing a pulse sequence which enhances visualization of flowing blood.)

In PET systems (TOF and non-TOF), a pair of detected photons is deemed coincident if the photons are detected "at the same time". The threshold for the difference in detection time that is considered "at the same time" depends on the timing accuracy of the PET detectors. For example, for a non-TOF PET system the timing accuracy may be on the order of 5000 picoseconds (ps). This coincident window, which is the acceptable range of the timing difference of detected events to be considered coincident, is set as small as possible to minimize random coincidence events arising from unrelated positron decays but is not set too small to inadvertently eliminate true coincidence events.

PET detectors operating in TOF mode are fast enough to resolve the location of the point of annihilation within some margin of error on the line of coincidence and to use this information during image reconstruction. The $511 \mathrm{keV}$ annihilation photons travel at the speed of light, so one photon interacts with its detector slightly earlier than the other photon does, unless the annihilation event occurs equidistant from the two detectors. Using the speed of light, one can calculate the spatial location on the coincidence line with precision dependent on the timing resolution of the PET detectors. Modern clinical PET detectors using lutetium-based crystals and PMTs have a timing resolution* that is better than $600 \mathrm{ps.}$ Given such timing, the site of annihilation can be spatially localized with precision better than $9 \mathrm{~cm}$ along the line connecting the crystals that detected the photons (i.e. the line of response). While the location is not precisely known, the image reconstruction localizes counts over this range, a distance that is significantly shorter than the diameter of the scanner. By utilizing this enhanced information, TOF scanners have reduced image noise compared to a non-TOF scanner, for an equal number of counts. ${ }^{47,} 48$ The improved PET performance from TOF may be exploited by shortening the scan duration and/or reducing the injected radiopharmaceutical activity to reduce radiation dose to the patient, while maintaining acceptable image quality.

Current sequential clinical PET/MR (and PET/CT) scanners use conventional PMT-based detectors that are TOF-capable, having full-width at half-maximum (FWHM) timing

\footnotetext{
*Timing and spatial localization here are specified according to the full-width at half-maximum (FWHM).
} 
resolution in the range of 500 to 600 picoseconds. At this time, simultaneous clinical PET/MR scanners use APD-based detectors which, although compatible with high magnetic fields, have a timing resolution of 3600 picoseconds. This timing resolution corresponds to a localization precision of $54 \mathrm{~cm}$ along the line of response, which is not appreciably smaller than the field of view. Consequently, APD-based detectors are not considered to be TOFcapable.

\section{D. Motion}

Patient motion is always a concern in medical imaging. In all cases measures are taken to minimize gross patient motion (foam pads, tape, etc.). However internal patient motion from bladder-filling, digestion, etc. also degrades images. Small movement is apparent as artifacts in MR images because of the high spatial resolution, whereas in PET images it tends to be noticed as increased blurring and reduced peak uptake of lesions. In combined PET/MR, a further effect of motion is in misregistration of PET and MR images. For the case of simultaneous PET/MR, the motion is "in-place", meaning that a portion of the PET data matches the MR data, thus mitigating the misregistration to some degree. For the case of sequential PET/MR, the data are less correlated, especially if there is a long period of time between the MR and PET acquisitions. (The situation is similar for PET/CT, though the time between $\mathrm{CT}$ and PET is short.) The one-room sequential PET/MR design minimizes this possibility since about a minute is required to reposition the patient on the scan table between MR mode and PET modes. The two-room sequential design has a greater possibility for motion to occur, since the patient must be unloaded from one table, transported to the other room, and reloaded onto the other table.

An interesting development for simultaneous PET/MR is to perform MR-based motion correction of PET data. While PET acquisition occurs for several minutes, MR is capable of rapidly producing images or motion tracking signal (navigator) to serve as an anatomic and temporal reference for the PET data. One could imagine the MR as taking a movie of the patient during PET scanning. The PET data handling could offset the position of individual photon coincidence pairs to account for the motion and thereby mitigate motion blurring. A group at Massachusetts General Hospital (MGH) first demonstrated this approach with a brain PET detector insert in a 3T MR scanner, showing accurate rigid-body motion correction with 3 second temporal precision. ${ }^{49}$ More recent work has shown the ability to measure motion deformation fields with MR and to apply this information towards non-rigid motion correction of simultaneously-acquired PET data. ${ }^{50-52}$

\section{E. Attenuation correction}

As described above, there are significant limitations associated with MR-based attenuation correction of PET data. It is first worth noting that tri-modality sequential PET/MR (that is, PET/CT co-registered to MR) utilizes CT-based attenuation correction and avoids the quantitative inaccuracies of MR-based attenuation correction. Tri-modality systems are valuable in MR-AC research since the same PET data are available for both CT-AC and MR-AC, allowing for a direct comparison of MR-AC to the CT-AC standard. ${ }^{53}$ 
With the exception of the tri-modality design, both sequential and simultaneous PET/MR do not have the ability to perform CT-AC, and thus the challenges of MR-AC cannot be avoided at this time. Ultimately there might be a solution for this that simultaneously reconstructs emission and transmission images solely using PET emission data. Researchers have found evidence that this may be feasible with TOF PET (see section 7.A).

\section{F. Complexity and amount of new engineering}

The engineering complexity varies significantly between the three PET/MR designs. The trimodality sequential PET/CT + MR system is the least complex, with new engineering residing primarily in the shuttle bed and docking system. The one-room sequential PET/MR system has significant new engineering in the turntable patient bed and in the modified PET gantry containing magnetic and electronic shielding. The acquisition workstation is also enhanced to acquire both PET and MR data and to perform MR-AC.

By far the most new engineering is in the simultaneous PET/MR system. New PET detectors with APDs are used for the first time in a commercial PET scanner. The magnet hardware and cryogenic system are redesigned to integrate the PET detectors in the field of view while allowing field service engineers access to the PET detectors and supporting electronics. Electronic shielding is a greater challenge due to the proximity of the PET and MR hardware, compared to the sequential design. Another major but often underappreciated engineering challenge is the software and firmware that allows all components to function in harmony in a fully integrated imaging system.

Of course, new engineering is associated with cost and reliability. An old engineering saying is: "better, faster, cheaper - pick no more than two out of the three". The PET/MR vendors have done a remarkable job in providing amazing new technology in such a short period of time. Economics, however, remains a major factor in clinical acceptance.

\section{The Future}

\section{A. New technologies}

Avalanche photodiodes (APDs) are more compact than photomultiplier tubes (PMTs) and perform the same function: converting the scintillation light created in the crystal into an electrical signal. A main disadvantage of APDs is that their timing precision is poor and cannot support time-of-flight measurement. A special type of APD, called a silicon photomultiplier (SiPM) overcomes this limitation. A SiPM consists of an array of many miniature APD cells each operating in Geiger mode, which means that the signal from each cell is all-or-nothing. The amount of scintillation light (and hence gamma-ray energy) is indicated by the number of cells that fire. Like APDs, SiPMs are compact and insensitive to magnetic fields but require cooling to reduce noise. However SiPMs have higher gain and good timing resolution and thus are suitable for TOF PET/MR. The timing resolution of fullring prototype PET detectors with lutetium-based crystals has been demonstrated to be better than 400 ps with analog SiPMs ${ }^{54}$ and better than 300 ps with digital SiPMs ${ }^{55}$. Recent work has suggested that timing resolution better than 200 ps may be feasible. ${ }^{56}$ With such improved timing resolution over PMT-based detectors, SiPMs are poised to make a significant impact on PET/CT as well as PET/MR, as availability and cost improve. In fact, 
Philips recently has announced a new PET/CT product (Vereos) using PET detectors with digital SiPMs, which marks a significant development in clinical PET technology.

Another exciting potential future development is TOF-assisted attenuation correction for PET/MR. It has long been a goal to use the attenuation information contained within the PET emission data alone to reconstruct both attenuation and emission images. ${ }^{57-59}$ However crosstalk between the attenuation and emission information results in a large degree of uncertainty in the images. Crosstalk arises from the fact that low activity and high attenuation along a path through the body both result in a low number of recorded counts. Recently it has been discovered that TOF provides extra information that greatly reduces the crosstalk problem, allowing for attenuation correction solely from the emission data. ${ }^{60-62}$ This method is intriguing for PET/MR because it avoids many challenges associated with MR-based attenuation correction. The method does not apply to current-generation simultaneous PET/MR using APDs which are not TOF-capable. It may one day apply to sequential PET/MR using PMTs already TOF-capable, as well as future generations of simultaneous PET/MR having SiPM detectors.

\section{B. New designs}

We already have seen three significantly different commercial PET/MR designs: trimodality sequential PET/CT + MR, one-room sequential PET/MR, and simultaneous PET/MR. A possible fourth design is analogous to PET/CT: an integrated sequential PET/MR. In this design, the PET detectors would be adjacent to the MR magnet, and the patient table would have extended travel to pass through both fields of view. A main advantage of this design is that the bore diameter is preserved. Thus a $70-\mathrm{cm}$ bore diameter would be available for both PET and MR. Alternatively, a less expensive magnet with 60$\mathrm{cm}$ bore diameter may be used to control cost. However an integrated sequential PET/MR would not be capable of simultaneous PET/MR acquisition since the PET and MR fields of view would not overlap.

Another design consideration is the quality of the MR system. The three commercial PET/MR systems all have used 3T magnets for optimal MR imaging performance. However this comes at a significantly higher cost compared to lower-end 1.5T magnets. Similarly, the cost of the PET detectors may be better contained by reducing the axial field of view, at the expense of sensitivity and image quality. Thus a relatively low-cost PET/MR system (integrated sequential with smaller bore size, field strength, and axial field of view) may be feasible if there is clinical demand for such a scanner.

\section{C. What is the clinical future of PET/MR?}

The main focus of our discussion has been on technology. Ultimately many other factors will determine the overall success of PET/MR in the clinical environment. Among these are cost, clinical impact, reimbursement, and competing technologies. There is certain to be a vibrant market for PET/MR in research and at academic medical centers. MR's exquisite image quality, soft tissue contrast, flexibility of pulse sequences, and advanced capabilities of spectroscopic and functional imaging offer unique opportunities compared to CT. 
How will PET/MR fare in the routine clinical environment? At the moment the PET/MR community is seeking to identify indispensable clinical applications that justify PET/MR, the so-called "killer apps".

Will breast cancer imaging be a killer-app? MR has exquisite sensitivity for staging breast cancer and is, perhaps, too sensitive as to have false positives and lead to unnecessary biopsies ${ }^{63}$; up to $75 \%$ turn out to be benign ${ }^{64}$. Will combining the specificity of PET and the sensitivity and spatial resolution of MR produce a winner? It may with PET/MR particularly considering that with separate PET/CT and MR, correlation is complicated due to the deformability of breast tissue.

Will radiation therapy planning be a killer-app? PET/CT has already attracted much interest for radiation therapy planning. Many centers utilize consistent patient positioning tools in their clinical workflows and incorporate PET/CT in their treatment plans. While CT is the workhorse in this arena, MR offers better soft tissue contrast and therefore might help to better pinpoint the radiation beam to cancer while sparing normal tissue in, for example, prostate cancer ${ }^{65-69}$. Indeed, there is growing interest in coupling radiation therapy's linear accelerators with MR scanners ${ }^{70-72}$.

Another potential killer-app is motion-tracking and improved PET quantification with truly simultaneous PET/MR scanning. Respiratory and cardiac motion causes some tumors to move during imaging that leads to blurring in the image. With knowledge of the motion from MR, the blurring could be removed during PET image reconstruction. Also, the high spatial resolution available with MR could also be used to mitigate blurring (and inaccuracies of image-derived radioactivity concentrations) of small objects due to partial volume effects. Of course the size and motion degradations are often not independent such as in the case of a small blood vessel. MR could provide the geometric information needed to accurately determine an image-derived input function that could be used to quantify blood flow to a tumor.

A benefit of PET/MR is reduced exposure to ionizing radiation compared to PET/CT. A typical whole-body PET/CT study using low-dose CT may have an effective radiation dose of $7 \mathrm{mSv}$ from the injected ${ }^{18} \mathrm{~F}-\mathrm{FDG}$ and $4 \mathrm{mSv}$ from the $\mathrm{CT}$. (The actual radiation dose differs between imaging centers depending on injected activity and CT parameters.) New technologies (improved TOF performance from SiPMs and improved CT image quality at lower x-ray intensity from iterative reconstruction) promise to further reduce radiation dose in PET/CT in the future. However, PET/MR eliminates the CT radiation which offers an immediate and sizable reduction in radiation dose. The increasing attention on radiation dose for pediatric patients and for patients receiving several follow-up PET/CT studies makes PET/MR attractive.

Another immediate clinical benefit of PET/MR is the ability to perform PET and MR in one session. Of course patient satisfaction is improved from the convenience of scheduling and undergoing a single study instead of two. More importantly, there are medical benefits in cases requiring sedation, more common for pediatric patients, for which PET/MR utilizes a 
single shorter sedation instead of multiple or longer sedations for separate PET and MR studies.

Ultimately, it likely will be economics that determine the success of PET/MR in the clinical environment. There are certain advantages of PET/MR compared to PET/CT, but will the benefits outweigh the added cost? In this era of increasing regulation and decreasing reimbursement, PET/MR will have to prove that clinical outcomes are economically superior to the alternative (PET/CT and MR acquired independently, potentially with software fusion). This argument will be easier if the cost of PET/MR systems drops as the market increases or as cost-reduction engineering proceeds. As discussed earlier, the rise of PET/CT and its rapid displacement of dedicated PET were truly remarkable. However the bar already has been set high by the success of PET/CT, so the challenge of widespread clinical acceptance is even higher for PET/MR. The next few years will be very interesting as data on the relative clinical value of PET/MR are reported.

\section{Supplementary Material}

Refer to Web version on PubMed Central for supplementary material.

\section{Acknowledgements}

The authors thank Lingzhi (Lance) Hu PhD and Andrew Sher MD for scientific review of the manuscript.

\section{References}

1. Moller AK, Loft A, Berthelsen AK, Pedersen KD, Graff J, Christensen CB, Costa JC, Skovgaard LT, Perell K, Petersen BL, Daugaard G. A prospective comparison of 18F-FDG PET/CT and CT as diagnostic tools to identify the primary tumor site in patients with extracervical carcinoma of unknown primary site. The oncologist. 2012; 17:1146-1154. [PubMed: 22711751]

2. Herzog H, Van Den Hoff J. Combined PET/MR systems: an overview and comparison of currently available options. Q J Nucl Med Mol Imaging. 2012; 56:247-267. [PubMed: 22695336]

3. Kalemis A, Delattre BM, Heinzer S. Sequential whole-body PET/MR scanner: concept, clinical use, and optimisation after two years in the clinic. The manufacturer's perspective. MAGMA. 2013; 26:5-23. [PubMed: 22868642]

4. Hasegawa BH, Gingold EL, Reilly SM, Liew S-C, Cann CE. Description of a simultaneous emission-transmission CT system. Proc SPIE. 1990; 1231:50-60.

5. Lang TF, Hasegawa BH, Liew SC, Brown JK, Blankespoor SC, Reilly SM, Gingold EL, Cann CE. Description of a prototype emission-transmission computed tomography imaging system. Journal of nuclear medicine : official publication, Society of Nuclear Medicine. 1992; 33:1881-1887.

6. Blankespoor SC, Wu X, Kalki K, Brown JK, Tang HR, Cann CE, Hasegawa BH. Attenuation correction of SPECT using X-ray CT on an emission-transmission CT system: myocardial perfusion assessment. IEEE Trans Nucl Sci. 1996; 43:2263-2274.

7. Patton JA, Townsend DW, Hutton BF. Hybrid imaging technology: from dreams and vision to clinical devices. Seminars in nuclear medicine. 2009; 39:247-263. [PubMed: 19497402]

8. Beyer T, Townsend DW, Brun T, Kinahan PE, Charron M, Roddy R, Jerin J, Young J, Byars L, Nutt R. A combined PET/CT scanner for clinical oncology. Journal of nuclear medicine : official publication, Society of Nuclear Medicine. 2000; 41:1369-1379.

9. Townsend DW. Positron emission tomography/computed tomography. Semin Nucl Med. 2008; 38:152-166. [PubMed: 18396176] 
10. Cohade C, Wahl RL. Applications of positron emission tomography/computed tomography image fusion in clinical positron emission tomography-clinical use, interpretation methods, diagnostic improvements. Semin Nucl Med. 2003; 33:228-237. [PubMed: 12931324]

11. Garlick PB, Marsden PK, Cave AC, Parkes HG, Slates R, Shao Y, Silverman RW, Cherry SR. PET and NMR dual acquisition (PANDA): applications to isolated, perfused rat hearts. NMR in biomedicine. 1997; 10:138-142. [PubMed: 9408923]

12. Marsden PK, Shao Y, Cherry SR, Cave A, Parkes HG, Silverman RW, Slates R, Meadors K, Bowery A, Garlick PB. Simultaneous acquisition of PET images and NMR spectra in a high field magnet. Journal of Nuclear Medicine. 1997; 38:161-161.

13. Shao Y, Cherry SR, Farahani K, Meadors K, Siegel S, Silverman RW, Marsden PK. Simultaneous PET and MR imaging. Physics in medicine and biology. 1997; 42:1965-1970. [PubMed: 9364592]

14. Shao Y, Cherry SR, Farahani K, Slates R, Silverman RW, Meadors K, Bowery A, Siegel S, Marsden PK, Garlick PB. Development of a PET detector system compatible with MRI/NMR systems. IEEE transactions on nuclear science. 1997; 44:1167-1171.

15. Beyer T, Townsend DW, Blodgett TM. Dual-modality PET/CT tomography for clinical oncology. Q J Nucl Med. 2002; 46:24-34. [PubMed: 12072843]

16. Townsend DW, Beyer T. A combined PET/CT scanner: the path to true image fusion. The British journal of radiology. 2002; 75(Spec No):S24-S30. [PubMed: 12519732]

17. Farahani K, Slates R, Shao Y, Silverman R, Cherry S. Contemporaneous positron emission tomography and MR imaging at $1.5 \mathrm{~T}$. Journal of magnetic resonance imaging : JMRI. 1999; 9:497-500. [PubMed: 10194724]

18. Melcher CL. Scintillation crystals for PET. Journal of nuclear medicine : official publication, Society of Nuclear Medicine. 2000; 41:1051-1055.

19. Lucas AJ, Hawkes RC, Ansorge RE, Williams GB, Nutt RE, Clark JC, Fryer TD, Carpenter TA. Development of a combined microPET-MR system. Technology in cancer research \& treatment. 2006; 5:337-341. [PubMed: 16866564]

20. Raylman RR, Majewski S, Lemieux SK, Velan SS, Kross B, Popov V, Smith MF, Weisenberger AG, Zorn C, Marano GD. Simultaneous MRI and PET imaging of a rat brain. Physics in medicine and biology. 2006; 51:6371-6379. [PubMed: 17148823]

21. Yamamoto S, Imaizumi M, Kanai Y, Tatsumi M, Aoki M, Sugiyama E, Kawakami M, Shimosegawa E, Hatazawa J. Design and performance from an integrated PET/MRI system for small animals. Annals of nuclear medicine. 2010; 24:89-98. [PubMed: 20058103]

22. Bindseil GA, Gilbert KM, Scholl TJ, Handler WB, Chronik BA. First image from a combined positron emission tomography and field-cycled MRI system. Magnetic resonance in medicine : official journal of the Society of Magnetic Resonance in Medicine / Society of Magnetic Resonance in Medicine. 2011; 66:301-305.

23. Boss A, Stegger L, Bisdas S, Kolb A, Schwenzer N, Pfister M, Claussen CD, Pichler BJ, Pfannenberg C. Feasibility of simultaneous PET/MR imaging in the head and upper neck area. European radiology. 2011; 21:1439-1446. [PubMed: 21308378]

24. Schlemmer HP, Pichler BJ, Schmand M, Burbar Z, Michel C, Ladebeck R, Jattke K, Townsend D, Nahmias C, Jacob PK, Heiss WD, Claussen CD. Simultaneous MR/PET imaging of the human brain: feasibility study. Radiology. 2008; 248:1028-1035. [PubMed: 18710991]

25. Judenhofer MS, Catana C, Swann BK, Siegel SB, Jung WI, Nutt RE, Cherry SR, Claussen CD, Pichler BJ. PET/MR images acquired with a compact MR-compatible PET detector in a 7-T magnet. Radiology. 2007; 244:807-814. [PubMed: 17709830]

26. Zaidi H, Ojha N, Morich M, Griesmer J, Hu Z, Maniawski P, Ratib O, Izquierdo-Garcia D, Fayad ZA, Shao L. Design and performance evaluation of a whole-body Ingenuity TF PET-MRI system. Physics in medicine and biology. 2011; 56:3091-3106. [PubMed: 21508443]

27. Delso G, Furst S, Jakoby B, Ladebeck R, Ganter C, Nekolla SG, Schwaiger M, Ziegler SI. Performance measurements of the Siemens mMR integrated whole-body PET/MR scanner. Journal of nuclear medicine : official publication, Society of Nuclear Medicine. 2011; 52:19141922. 
28. Veit-Haibach P, Kuhn FP, Wiesinger F, Delso G, von Schulthess G. PET-MR imaging using a trimodality PET/CT-MR system with a dedicated shuttle in clinical routine. MAGMA. 2013; 26:2535. [PubMed: 23053712]

29. Bushong, SC. Magnetic resonance imaging : physical and biological principles. St. Louis: Mosby; 1988.

30. Weirich C, Brenner D, Scheins J, Besancon E, Tellmann L, Herzog H, Shah NJ. Analysis and correction of count rate reduction during simultaneous MR-PET measurements with the BrainPET scanner. IEEE transactions on medical imaging. 2012; 31:1372-1380. [PubMed: 22374353]

31. Hammer BE, Christensen NL, Heil BG. Use of a magnetic field to increase the spatial resolution of positron emission tomography. Medical physics. 1994; 21:1917-1920. [PubMed: 7700198]

32. Moses WW. Fundamental Limits of Spatial Resolution in PET. Nuclear instruments \& methods in physics research. Section A, Accelerators, spectrometers, detectors and associated equipment. 2011; 648(Supplement 1):S236-S240.

33. Zaidi H, Ojha N, Morich M, Griesmer J, Hu Z, Maniawski P, Ratib O, Izquierdo-Garcia D, Fayad ZA, Shao L. Design and performance evaluation of a whole-body Ingenuity TF PET-MRI system. Phys Med Biol. 2011; 56:3091-3106. [PubMed: 21508443]

34. Schenck JF. The role of magnetic susceptibility in magnetic resonance imaging: MRI magnetic compatibility of the first and second kinds. Med Phys. 1996; 23:815-850. [PubMed: 8798169]

35. Yamamoto S, Kuroda K, Senda M. Scintillator selection for MR-compatible gamma detectors. IEEE Trans Nucl Sci. 2003; 50:1683-1685.

36. Delso G, Furst S, Jakoby B, Ladebeck R, Ganter C, Nekolla SG, Schwaiger M, Ziegler SI. Performance measurements of the Siemens mMR integrated whole-body PET/MR scanner. Journal of nuclear medicine : official publication, Society of Nuclear Medicine. 2011; 52:19141922.

37. Marriott CJ, Cadorette JE, Lecomte R, Scasnar V, Rousseau J, van Lier JE. High-resolution PET imaging and quantitation of pharmaceutical biodistributions in a small animal using avalanche photodiode detectors. Journal of nuclear medicine : official publication, Society of Nuclear Medicine. 1994; 35:1390-1396.

38. Pichler BJ, Judenhofer MS, Catana C, Walton JH, Kneilling M, Nutt RE, Siegel SB, Claussen CD, Cherry SR. Performance test of an LSO-APD detector in a 7-T MRI scanner for simultaneous PET/MRI. Journal of nuclear medicine : official publication, Society of Nuclear Medicine. 2006; 47:639-647.

39. Wehrl HF, Judenhofer MS, Wiehr S, Pichler BJ. Pre-clinical PET/MR: technological advances and new perspectives in biomedical research. European journal of nuclear medicine and molecular imaging. 2009; 36(Suppl 1):S56-S68. [PubMed: 19194703]

40. Berker Y, Franke J, Salomon A, Palmowski M, Donker HC, Temur Y, Mottaghy FM, Kuhl C, Izquierdo-Garcia D, Fayad ZA, Kiessling F, Schulz V. MRI-based attenuation correction for hybrid PET/MRI systems: a 4-class tissue segmentation technique using a combined ultrashortecho-time/Dixon MRI sequence. Journal of nuclear medicine : official publication, Society of Nuclear Medicine. 2012; 53:796-804.

41. Catana C, van der Kouwe A, Benner T, Michel CJ, Hamm M, Fenchel M, Fischl B, Rosen B, Schmand M, Sorensen AG. Toward implementing an MRI-based PET attenuation-correction method for neurologic studies on the MR-PET brain prototype. Journal of nuclear medicine : official publication, Society of Nuclear Medicine. 2010; 51:1431-1438.

42. Keereman V, Fierens Y, Broux T, De Deene Y, Lonneux M, Vandenberghe S. MRI-based attenuation correction for PET/MRI using ultrashort echo time sequences. Journal of nuclear medicine : official publication, Society of Nuclear Medicine. 2010; 51:812-818.

43. Dixon WT. Simple proton spectroscopic imaging. Radiology. 1984; 153:189-194. [PubMed: 6089263]

44. Bailey DL, Roach PJ, Bailey EA, Hewlett J, Keijzers R. Development of a cost-effective modular SPECT/CT scanner. Eur J Nucl Med Mol Imaging. 2007; 34:1415-1426. [PubMed: 17372731]

45. Judenhofer MS, Wehrl HF, Newport DF, Catana C, Siegel SB, Becker M, Thielscher A, Kneilling M, Lichy MP, Eichner M, Klingel K, Reischl G, Widmaier S, Rocken M, Nutt RE, Machulla HJ, 
Uludag K, Cherry SR, Claussen CD, Pichler BJ. Simultaneous PET-MRI: a new approach for functional and morphological imaging. Nature medicine. 2008; 14:459-465.

46. Martinez-Moller A, Eiber M, Nekolla SG, Souvatzoglou M, Drzezga A, Ziegler S, Rummeny EJ, Schwaiger M, Beer AJ. Workflow and Scan Protocol Considerations for Integrated Whole-Body PET/MRI in Oncology. Journal of nuclear medicine : official publication, Society of Nuclear Medicine. 2012; 53:1415-1426.

47. Karp JS, Surti S, Daube-Witherspoon ME, Muehllehner G. Benefit of Time-of-Flight in PET: Experimental and Clinical Results. J Nucl Med. 2008; 49:462-470. [PubMed: 18287269]

48. Schaefferkoetter J, Casey M, Townsend D, El Fakhri G. Clinical impact of time-of-flight and point response modeling in PET reconstructions: a lesion detection study. Physics in Medicine and Biology. 2013; 58:1465-1478. [PubMed: 23403399]

49. Catana C, Benner T, van der Kouwe A, Byars L, Hamm M, Chonde DB, Michel CJ, El Fakhri G, Schmand M, Sorensen AG. MRI-assisted PET motion correction for neurologic studies in an integrated MR-PET scanner. Journal of nuclear medicine : official publication, Society of Nuclear Medicine. 2011; 52:154-161.

50. Chun SY, Reese TG, Ouyang J, Guerin B, Catana C, Zhu X, Alpert NM, El Fakhri G. MRI-based nonrigid motion correction in simultaneous PET/MRI. Journal of nuclear medicine : official publication, Society of Nuclear Medicine. 2012; 53:1284-1291.

51. King AP, Buerger C, Tsoumpas C, Marsden PK, Schaeffter T. Thoracic respiratory motion estimation from MRI using a statistical model and a 2-D image navigator. Medical image analysis. 2012; 16:252-264. [PubMed: 21959365]

52. Dikaios N, Izquierdo-Garcia D, Graves MJ, Mani V, Fayad ZA, Fryer TD. MRI-based motion correction of thoracic PET: initial comparison of acquisition protocols and correction strategies suitable for simultaneous PET/MRI systems. European radiology. 2012; 22:439-446. [PubMed: 21938440]

53. Samarin A, Burger C, Wollenweber SD, Crook DW, Burger IA, Schmid DT, von Schulthess GK, Kuhn FP. PET/MR imaging of bone lesions--implications for PET quantification from imperfect attenuation correction. European journal of nuclear medicine and molecular imaging. 2012; 39:1154-1160. [PubMed: 22526955]

54. Kim, C.; McDaniel, D.; Malaney, J.; McBroom, G.; Peterson, W.; Tran, VH.; Guo, J.; Ivan, A.; Dolinsky, S.; Wagadarikar, A.; Byun, A.; Fu, G.; Ganin, A. Time-of-flight PET-MR detector development with silicon photomultiplier; 2012 IEEE Nuclear Science Symposium Conference Record; 2012. p. 3533-3536.

55. Degenhardt, C.; Rodrigues, P.; Trindade, A.; Zwaans, B.; Mulhens, O.; Dorscheid, R.; Thon, A.; Salomon, A.; Frach, T. Performance evaluation of a prototype positron emission tomography scanner using digital photon counters (DPC); 2012 IEEE Nuclear Science Symposium Conference Record; 2012. p. 2820-2824.

56. Yeom JY, Vinke R, Levin CS. Optimizing timing performance of silicon photomultiplier-based scintillation detectors. Physics in Medicine and Biology. 2013; 58:1207-1220. [PubMed: 23369872]

57. Clinthorne NH, Fessler JA, Hutchins GD, Rogers WL. Joint maximum likelihood estimation of emission and attenuation densities in PET. IEEE Nuclear Science Symposium Conference Record. 1991; 3:1927-1932.

58. Erdogan H, Fessler JA. Joint estimation of attenuation and emission images from PET scans. IEEE Nuclear Science Symposium Conference Record. 1999; 3:1672-1675.

59. Glatting G, Wuchenauer M, Reske SN. Simultaneous iterative reconstruction for emission and attenuation images in positron emission tomography. Medical Physics. 2000; 27:2065-2071. [PubMed: 11011734]

60. Salomon A, Goedicke A, Schweizer B, Aach T, Schulz V. Simultaneous reconstruction of activity and attenuation for PET/MR. IEEE transactions on medical imaging. 2011; 30:804-813. [PubMed: 21118768]

61. Defrise M, Rezaei A, Nuyts J. Time-of-flight PET data determine the attenuation sinogram up to a constant. Physics in Medicine and Biology. 2012; 57:885-899. [PubMed: 22290428] 
62. Rezaei A, Defrise M, Bal G, Michel C, Conti M, Watson C, Nuyts J. Simultaneous reconstruction of activity and attenuation in time-of-flight PET. IEEE transactions on medical imaging. 2012; 31:2224-2233. [PubMed: 22899574]

63. Berg WA, Madsen KS, Schilling K, Tartar M, Pisano ED, Larsen LH, Narayanan D, Ozonoff A, Miller JP, Kalinyak JE. Breast cancer: comparative effectiveness of positron emission mammography and MR imaging in presurgical planning for the ipsilateral breast. Radiology. 2011; 258:59-72. [PubMed: 21076089]

64. Adler LP, Weinberg IN, Bradbury MS, Levine EA, Lesko NM, Geisinger KR, Berg WA, Freimanis RI. Method for combined FDG-PET and radiographic imaging of primary breast cancers. The breast journal. 2003; 9:163-166. [PubMed: 12752623]

65. Loeffelbein DJ, Souvatzoglou M, Wankerl V, Martinez-Moller A, Dinges J, Schwaiger M, Beer AJ. PET-MRI fusion in head-and-neck oncology: current status and implications for hybrid PET/ MRI. Journal of oral and maxillofacial surgery : official journal of the American Association of Oral and Maxillofacial Surgeons. 2012; 70:473-483.

66. Boss A, Bisdas S, Kolb A, Hofmann M, Ernemann U, Claussen CD, Pfannenberg C, Pichler BJ, Reimold M, Stegger L. Hybrid PET/MRI of intracranial masses: initial experiences and comparison to PET/CT. Journal of nuclear medicine : official publication, Society of Nuclear Medicine. 2010; 51:1198-1205.

67. Souvatzoglou M, Eiber M, Martinez-Moeller A, Furst S, Holzapfel K, Maurer T, Ziegler S, Nekolla S, Schwaiger M, Beer AJ. PET/MR in prostate cancer: technical aspects and potential diagnostic value. European journal of nuclear medicine and molecular imaging. 2013; 40(Suppl 1):S79-S88. [PubMed: 23703457]

68. Souvatzoglou M, Eiber M, Takei T, Furst S, Maurer T, Gaertner F, Geinitz H, Drzezga A, Ziegler S, Nekolla SG, Rummeny EJ, Schwaiger M, Beer AJ. Comparison of integrated whole-body [(11)C]choline PET/MR with PET/CT in patients with prostate cancer. European journal of nuclear medicine and molecular imaging. 2013; 40:1486-1499. [PubMed: 23817684]

69. Arce-Calisaya P, Souvatzoglou M, Eiber M, Beer A, Scheidhauer K, Geinitz H, Schwaiger M. Sensitivity of PET/MRI to detect recurrence of prostate cancer. European journal of nuclear medicine and molecular imaging. 2013; 40:799. [PubMed: 23429932]

70. Smit K, van Asselen B, Kok JG, Aalbers AH, Lagendijk JJ, Raaymakers BW. Towards reference dosimetry for the MR-linac: magnetic field correction of the ionization chamber reading. Physics in medicine and biology. 2013; 58:5945-5957. [PubMed: 23938362]

71. Raaymakers BW, de Boer JC, Knox C, Crijns SP, Smit K, Stam MK, van den Bosch MR, Kok JG, Lagendijk JJ. Integrated megavoltage portal imaging with a 1.5 T MRI linac. Physics in medicine and biology. 2011; 56:N207-N214. [PubMed: 21934191]

72. Lagendijk JJ, Raaymakers BW, Raaijmakers AJ, Overweg J, Brown KJ, Kerkhof EM, van der Put RW, Hardemark B, van Vulpen M, van der Heide UA. MRI/linac integration. Radiotherapy and oncology : journal of the European Society for Therapeutic Radiology and Oncology. 2008; 86:25-29. [PubMed: 18023488] 


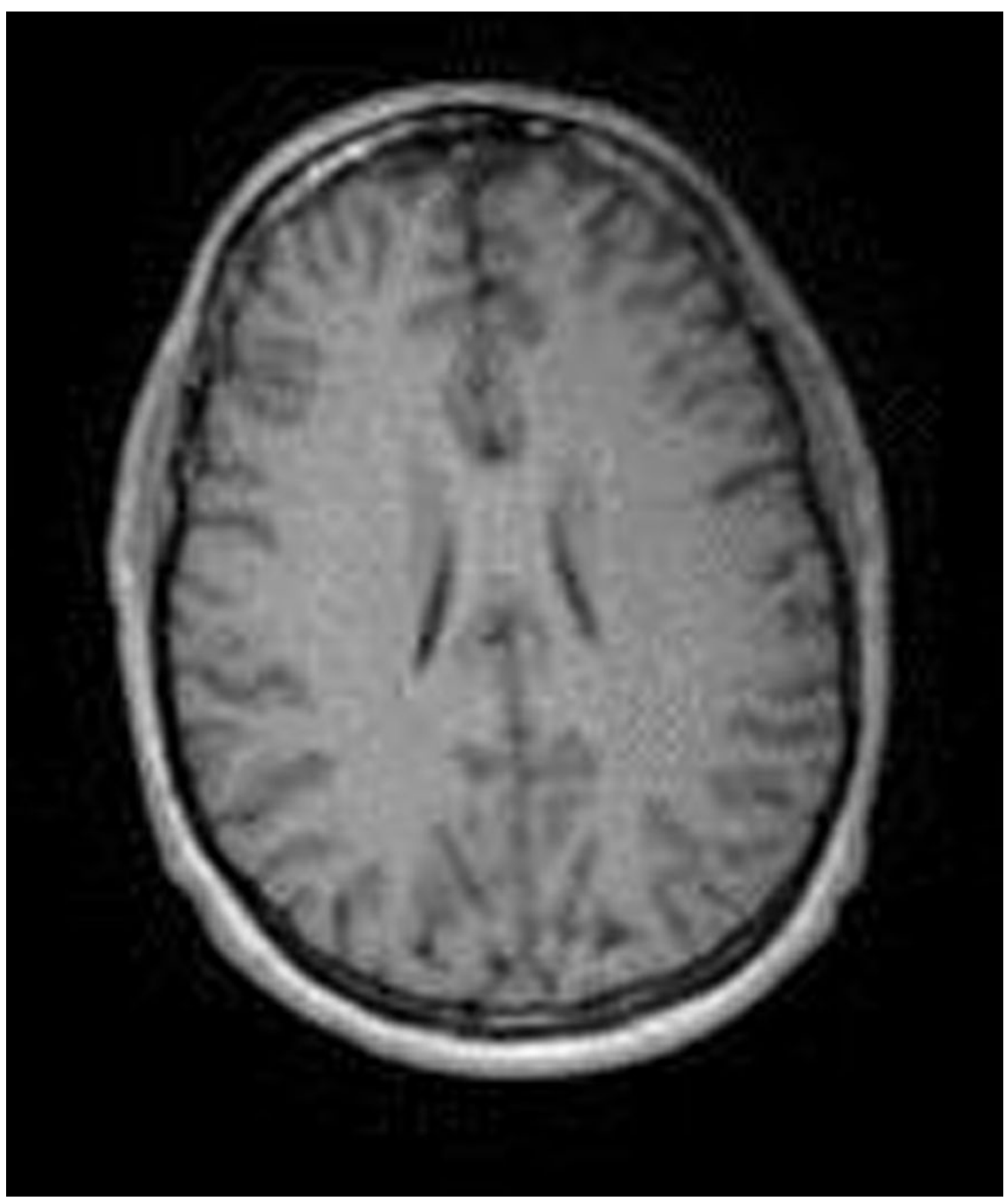

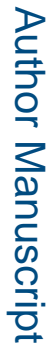




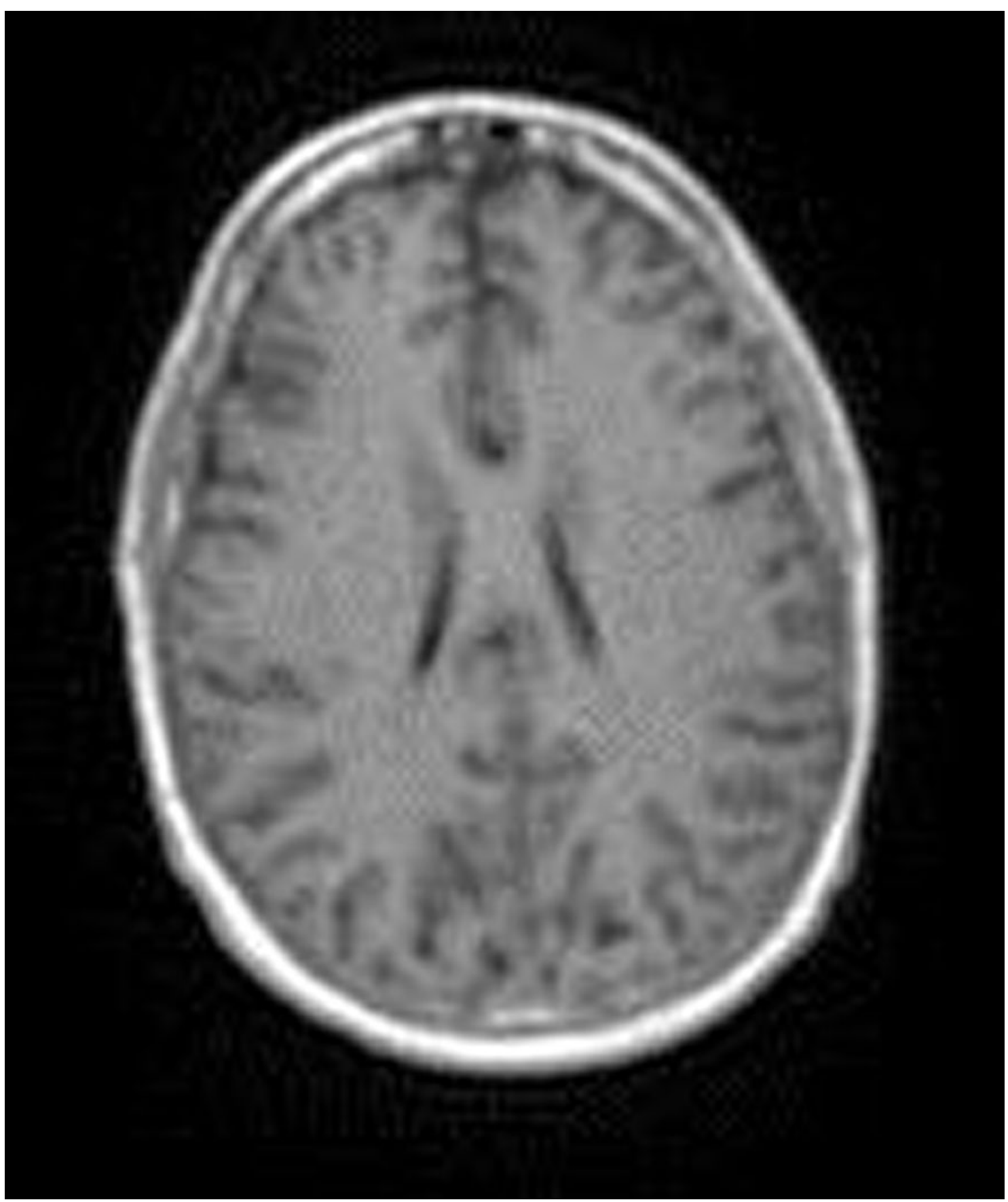

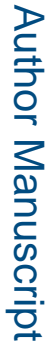




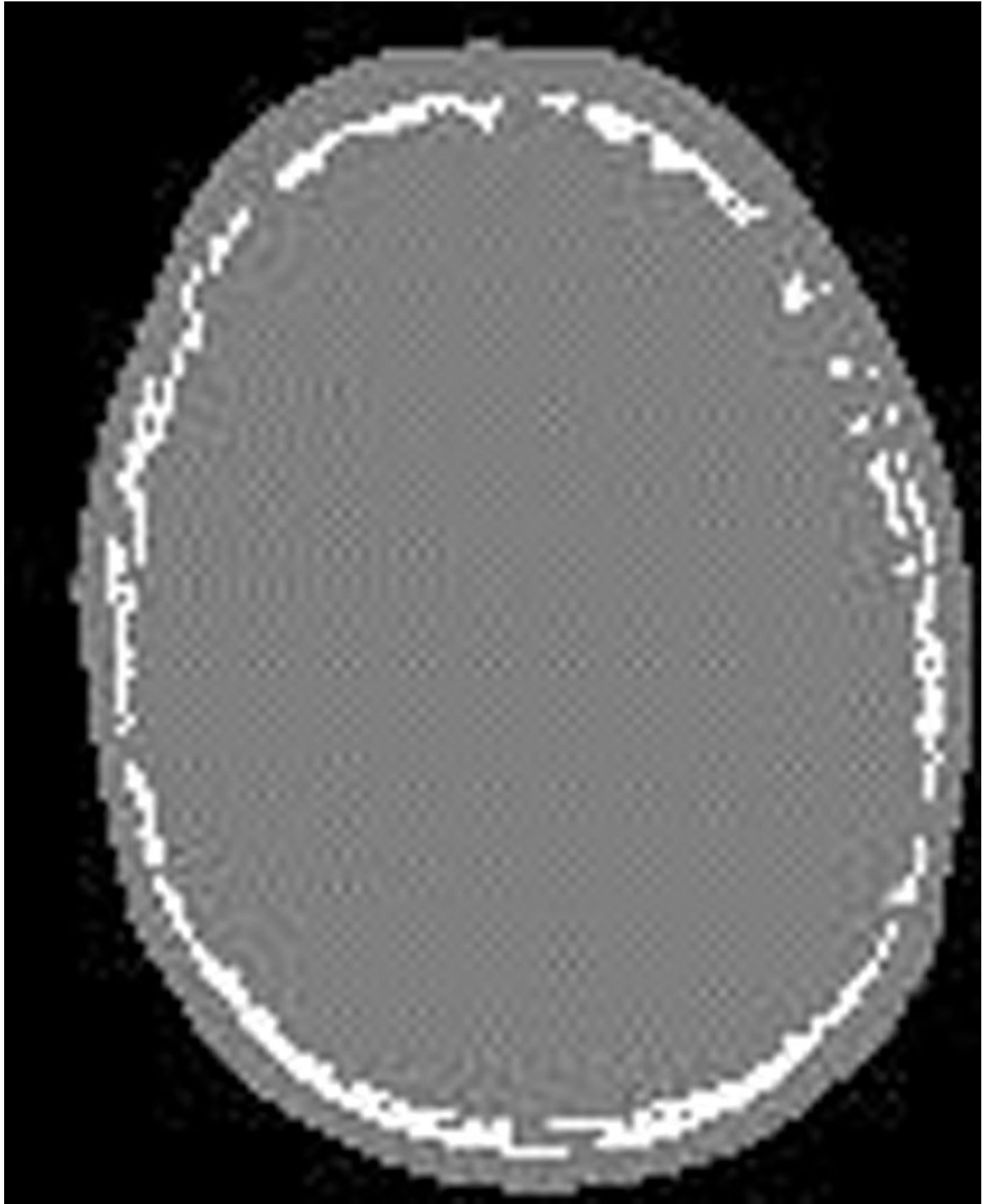

Figure 1.

With most MR imaging sequences, bone and air both have low signal and appear black. (a) In this axial image of the head, the skull is in the dark ring just outside the brain [TE $=2.46$ milliseconds (ms)]. (b) Using an ultra-short echo time [TE $=0.070 \mathrm{~ms}$ ] the data are collected before $\mathrm{T} 2 *$ relaxation completes and thus the bone of the skull has appreciable signal and appears gray. (c) Image processing methods may be applied to the first two images to produce an attenuation map that accounts for bone (white) and soft tissue (gray). 


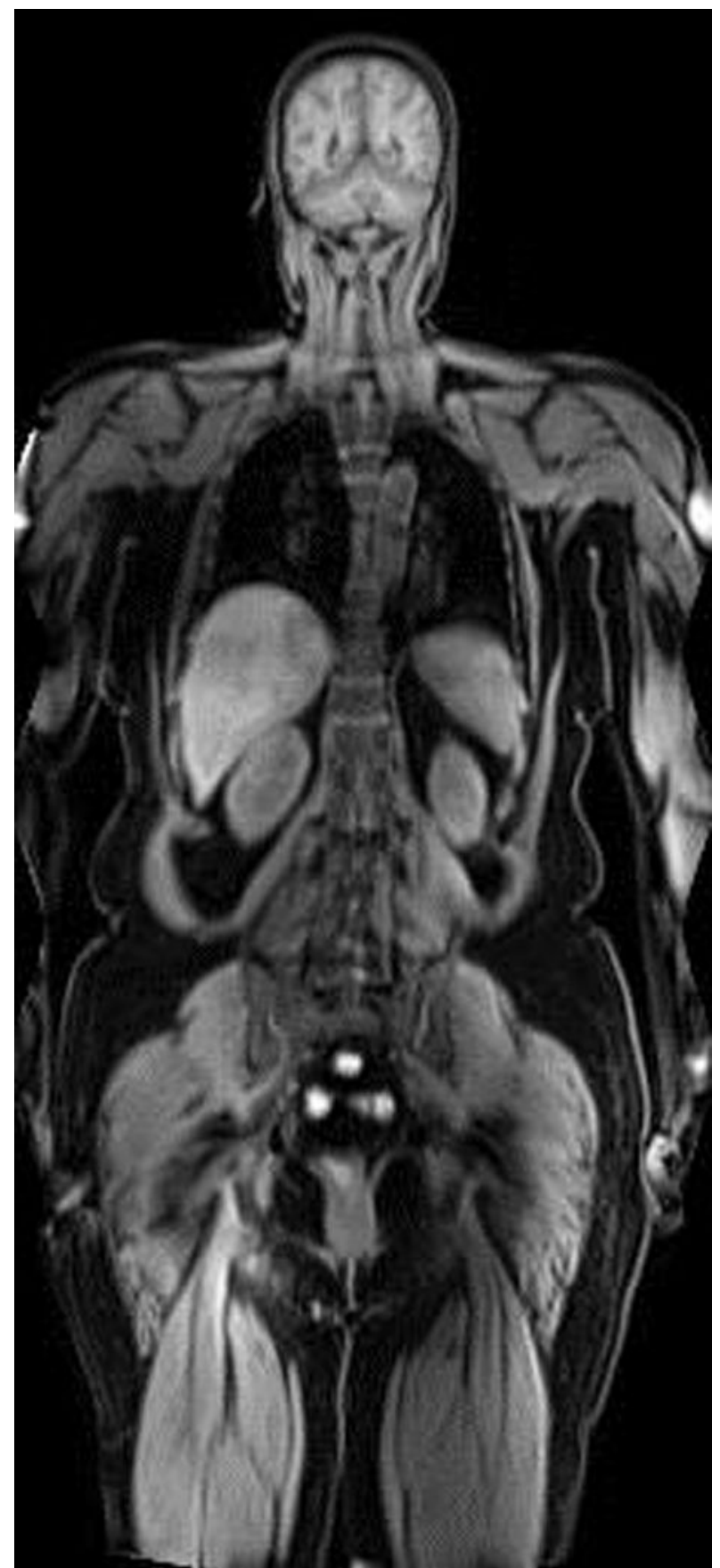

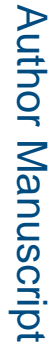

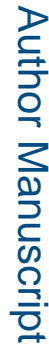

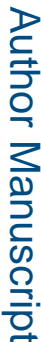




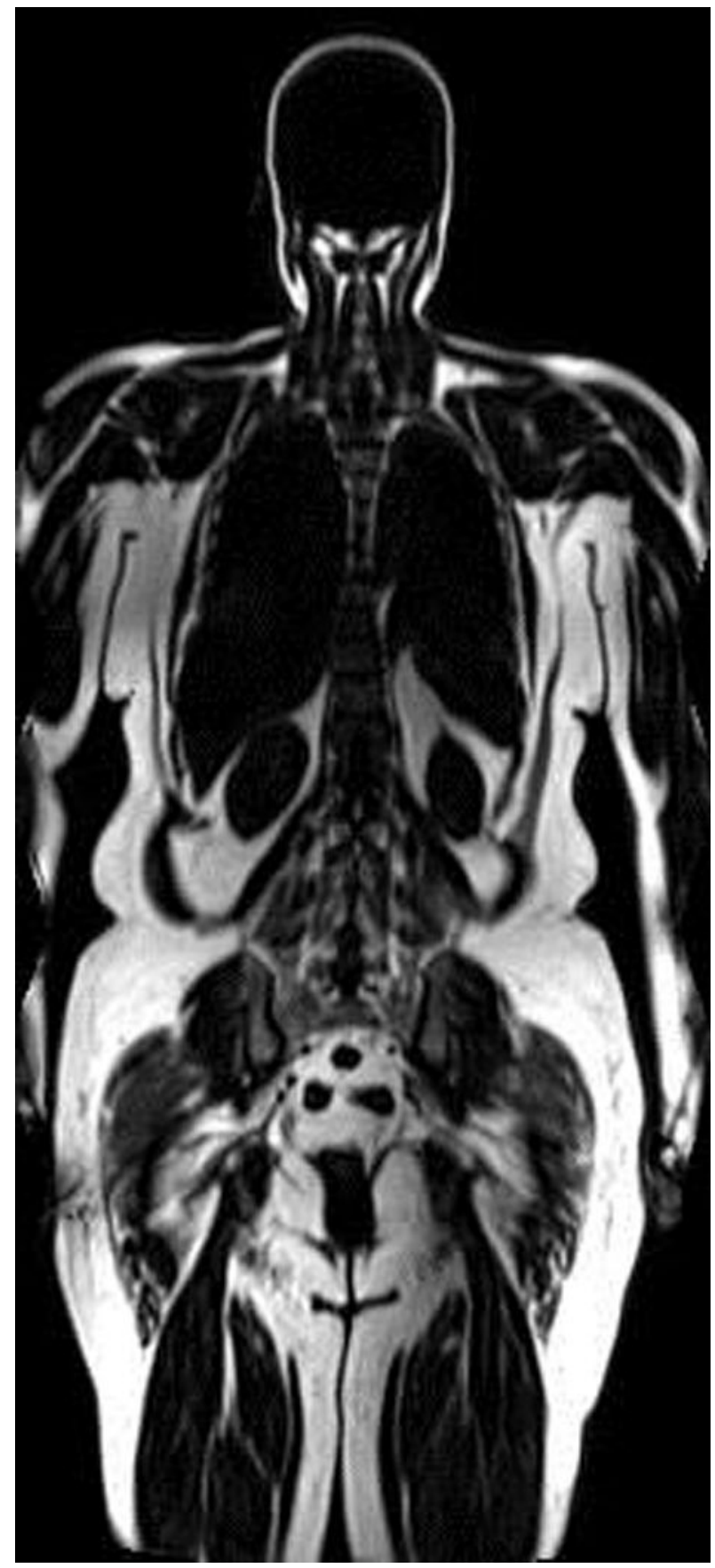

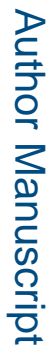




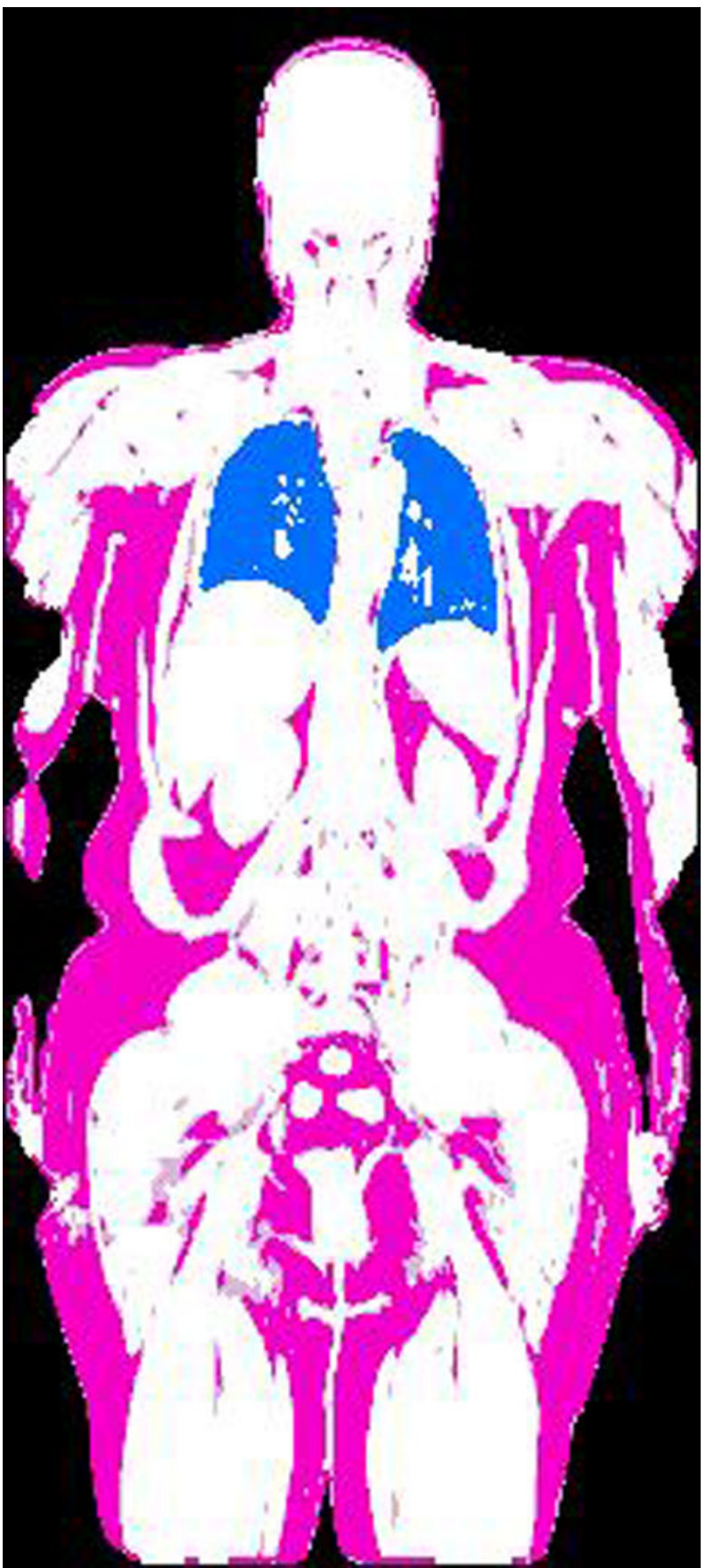

Figure 2.

MR-AC data generated by the two-point Dixon method. From left to right: a) water image, b) fat image, and c) segmented four-class attenuation map (lean tissue $=$ white, adipose tissue $=$ pink, lung $=$ blue, air $=$ black $)$. Note that the automated algorithm does not attempt to identify bone (given lack of bone information in Dixon) and defaults to treating it as water. 

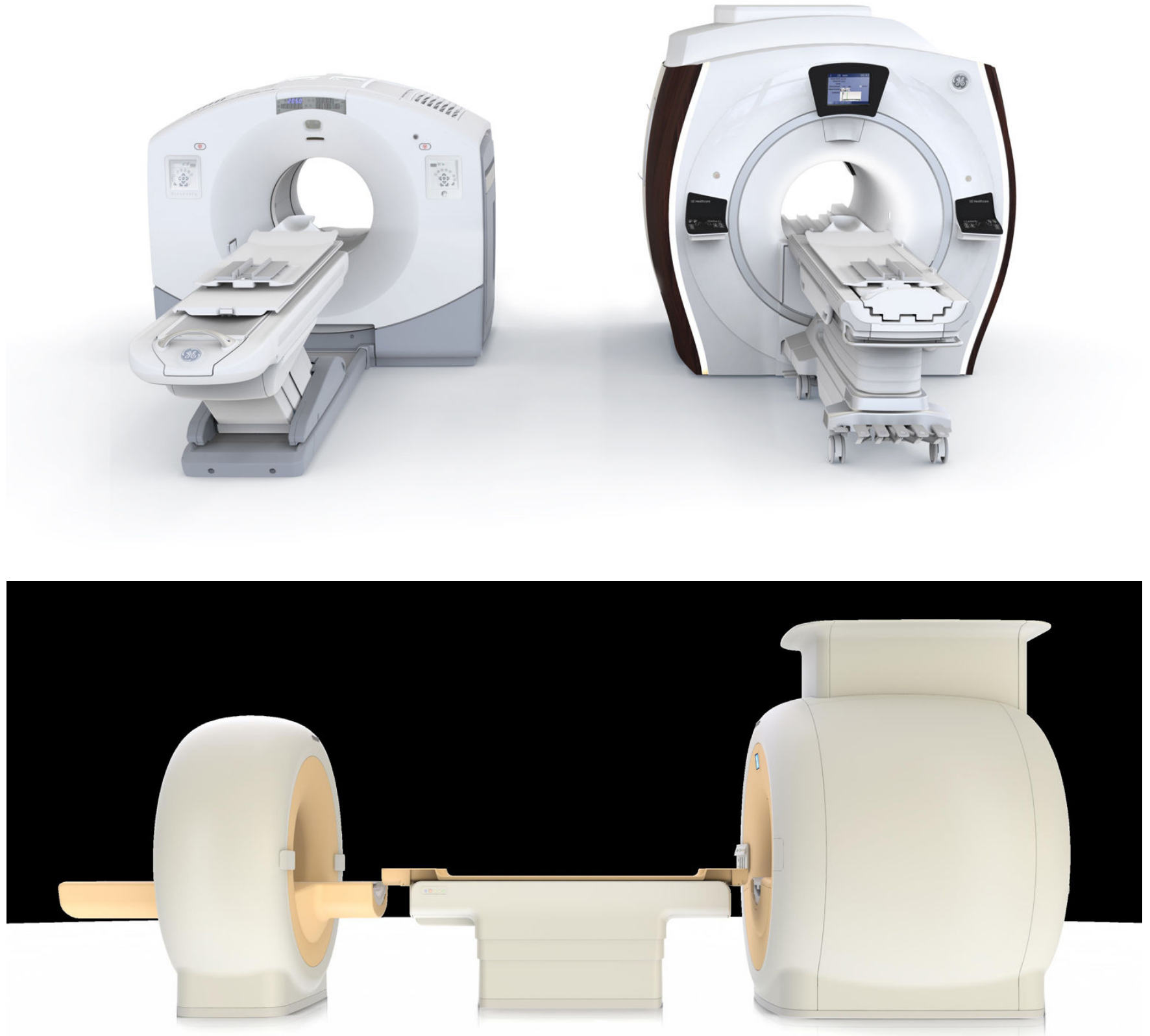

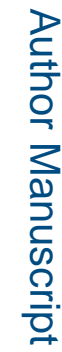




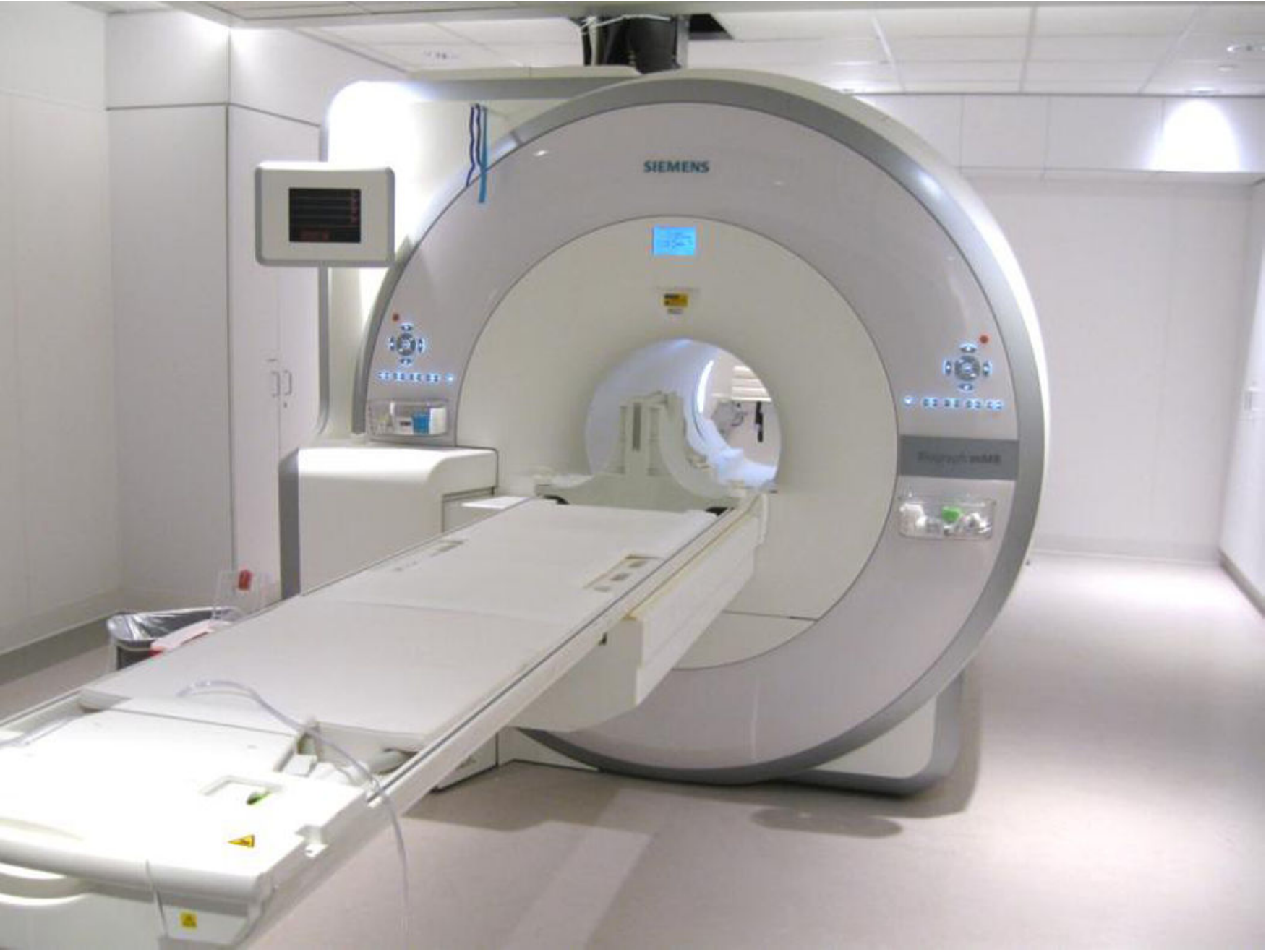

Figure 3 .

Photographs of (a) GE PET/CT+MR, (b) Philips Ingenuity TF PET/MR, and (c) Siemens Biograph mMR.

(a) Photo Courtesy of GE Healthcare

(b) Photo Courtesy of Philips Healthcare 


\section{PET WB multi-bed}

Figure 4.

Illustration of an example simultaneous whole-body (WB) PET/MR study. PET and MR WB data are acquired at multiple bed positions as shown. In this example three MR sequences are acquired at each bed position, each designated by a different color. After the WB scans are complete, an optional single-bed MR study may be acquired as in this example with two lengthy pulse sequences. Single-bed PET data (not shown) also may be acquired simultaneously with the single-bed MR study but will not be included in the WB images. 